\title{
User QoS-based Multi-Channel Assignment Schemes under Multimedia Traffic Conditions
}

\author{
Javier Gozálvez ${ }^{\#}, M^{a}$ Carmen Lucas Estañ ${ }^{\#}$ Joaquín Sánchez Soriano* \\ \#Signal Theory and Communications Division \\ *Operations Research Center \\ University Miguel Hernández, Avenida de la Univesidad s/n, 03202 Elche, Spain \\ j.gozalvez@umh.es
}

\begin{abstract}
The implementation of multi-channel assignment policies can improve a mobile's network performance by increasing the user throughput and reducing transmission delays. However, to define efficient distribution policies, the varying service demands and the search for user QoS fairness should be considered under multimedia traffic scenarios. In this context, this paper proposes and evaluates novel bankruptcy and utility-based multi-channel distribution schemes aimed at maximising the resource's efficiency and guaranteeing user QoS fairness.
\end{abstract}

\section{INTRODUCTION}

The increased appearance and acceptance of high bandwidth multimedia applications is creating new challenges to mobile operators in order to efficiently use the scarce available radio resources. In this context, various Radio Resource Management (RRM) policies have been defined and evaluated over the last years. One of these techniques is multichannel assignment, where different radio resources (time slots, codes, transmission power, etc) can be simultaneously and dynamically assigned to a given user.

While the assignment of several channels to a single user can increase its throughput and reduce its transmission delay, several key factors should be considered when implementing multi-channel assignment policies. First of all, the computational costs of implementing real-time channel assignment schemes should be clearly evaluated. In fact, the work reported in [1] showed that multi-channel distributions schemes that consider all possible distribution patterns result in excessive computational costs that prevent their use in current mobile communications systems. The impact of resource distribution policies under saturated mobile radio networks should also be investigated in order to define the appropriate policy's configuration under various traffic loads. Finally, it is also important to note that different traffic services might not require the same amount of radio resources to achieve a certain user QoS satisfaction level. Consequently, the design of multi-channel distribution schemes should consider user QoS fairness in heterogeneous traffic scenarios.

In this context, various multi-channel resource distribution policies have been reported in the literature. For example, the work reported in [2] proposed service-dependent weighted round robin scheduling policies and demonstrated the need to adapt the service user weight on the system load. An example of a channel assignment scheme seeking to fairly distribute available resources can be found in [3]. This proposal first estimates the user needed bandwidth to achieve certain QoS levels. Based on such estimates, the reported scheme equally distributes resources among users. However, it is important to note that the work in [3] distributes continuous and infinitively divisible frequency bands. On the other hand, this work focuses on multi-channel assignment policies where radio resources (time slots or codes) are discrete. Recent contributions have also proposed the use of economics concepts to address the multi-channel assignment problem. For example, [4] employs Vickrey auctions where the price users are willing to pay for radio resources is considered in the channel distribution policy.

This paper further expands the research on the potential of economics concepts to address the multi-channel assignment issue in heterogeneous traffic scenario by investigating the use of bankruptcy theories. In fact, given that in bankruptcy situations the value of a company is inferior to the sum of its debts, adequate distribution policies have to be defined to divide its net value among its creditors [5]. By exploiting this situation's similarity with the multi-channel assignment dilemma in highly loaded heterogeneous traffic environments, this work proposes a bankruptcy-based multi-channel assignment scheme. The proposal's performance is compared to that achieved with fixed assignment schemes and with mechanisms assigning resources based on the search for the maximum resource's system utilisation.

\section{ChanNEl ASSignMent POLICIES}

This work considers an heterogeneous traffic scenario where each traffic class has different QoS requirements. In this context, the proposed schemes are aimed at exploiting these varying resource needs to maximise channel efficiency and user QoS satisfaction levels. To achieve these objectives, it is important to first identify the resources needed per traffic class to achieve various QoS levels. Once such requirements have been identified, the bankruptcy proposal will be designed to maximise user QoS satisfaction fairness.

\section{A. Traffic utility values}

The proposed multi-channel assignment policies are based on the definition of adequate traffic class utility functions. This work considers an heterogeneous traffic scenario with email (background), WWW (interactive) and real-time H.263 video (at 16, 32 and $64 \mathrm{kbps}$ mean bit rates) transmissions. Following 3GPP recommendations [6], email and WWW 
transmissions are considered satisfactory if an email or web page is transmitted in less than 4 seconds. Real-time video transmissions are considered satisfactory if video frames are completely transmitted before the next video frame is to be transmitted. The utility functions employed in this work have been established defining minimum, mean and maximum user QoS satisfaction levels (see Table I). These QoS levels are based on user throughput for web and email transmissions, and on the percentage of correctly transmitted frames for realtime video services. The process to define such utility values has been based on extensive system level simulations. Due to space constraints, the paper focuses on the definition of the channel assignment policies rather than on the process followed to define the utility functions used by the policies. However, the results presented in this paper will validate the utility values selected to configure the proposed algorithms.

TABLE I

USER QOS LEVELS

\begin{tabular}{|c|c|c|c|}
\hline & Min. QoS & Mean QoS & Max. QoS \\
\hline WWW & $32 \mathrm{kbps}$ & $64 \mathrm{kbps}$ & $128 \mathrm{kbps}$ \\
\hline Email & $16 \mathrm{kbps}$ & $32 \mathrm{kbps}$ & $64 \mathrm{kbps}$ \\
\hline H.263 video & $75 \%$ & $95 \%$ & $100 \%$ \\
\hline Established utility values & $0.95 / 4$ & $0.95 / 2$ & 0.95 \\
\hline
\end{tabular}

Fig. 1 shows the utility values employed per traffic service and number of radio resources assigned to a user. As it will be later explained, this work is based on the EDGE (Enhanced Data Rate for GSM Evolution) radio interface which allows to assign multiple channels (in this case time slots) to a single user. As a result, the radio resources to be assigned correspond to time slots (TS), and each user can be assigned a maximum eight time slots. It is important to note that EDGE dynamically varies the used transmission mode (modulation and coding scheme) based on the channel quality variations. Given it is not possible to predict beforehand such channel quality variations, the utility values reported in Fig. 1 were obtained considering the use of a fixed EDGE transmission mode (in this case MCS5 which provides a mean bit rate of $22.4 \mathrm{kbps}$ per timeslot and is based on 8PSK. This choice was made after extensive EDGE system level simulations that identified MCS5 as the most widely used transmission mode

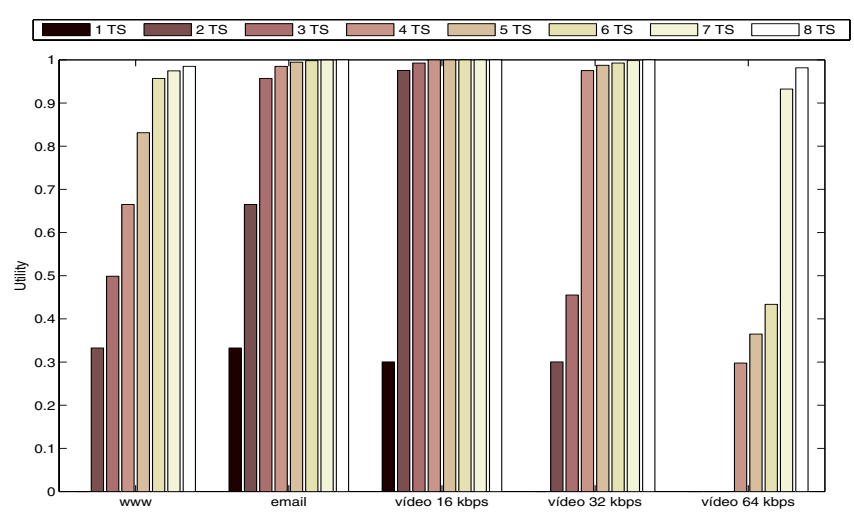

Fig. 1. Utility values per traffic service and number of assigned TS under the considered operating conditions. Although the operating conditions have been maintained in this paper, different system configurations could require the redefinition of the utility values represented in Fig. 1.

It is important to note from Fig. 1 that certain traffic services require more than one time slot to achieve utility values greater than zero. This is the case because the proposed bankruptcy scheme has been designed to try to always guarantee the minimum QoS level defined in Table I, and certain traffic services need more than one time slot to achieve such minimum QoS.

\section{B. Discrete CEL (DCELM)}

Several bankruptcy policies have been developed to distribute a limited number of resources among a large number of creditors [5]. An interesting option that could be adapted to guarantee user QoS fairness in mobile radio networks is Constraint Equal Loss (CEL). CEL operation is defined considering a value $E \in R_{+}$that has to be divided among $N$ agents whose value is greater than $E$. For each $i \in N$, $c_{i} \in R_{+}$represents the resource's request by $i$, and $c \equiv\left(c_{i}\right)_{i \in N}$ the requests vector. In this context, a resources distribution dilemma is denoted by the pair $(c, E) \in R_{+} \times R_{+}$, such that $\Sigma c_{i} \geq$ $E$. CEL considers that initially each agent has been assigned its maximum resources request. Given that such requests cannot be fulfilled in loaded systems, CEL equally reduces the amount of resources to each agent; a negative number of resources is not a viable solution. The CEL distribution rule can be defined as: $C E L(c, E) \equiv \max \{0, c-\lambda\}$, where $\lambda$ is chosen so that $\Sigma \max \{0, c-\lambda\}=E$. CEL adds the amount of resources requested per user to achieve maximum user satisfaction and computes the difference between this value and the amount of available radio resources $\Sigma c_{i}-E$. The resulting value is equally divided between the $\mathrm{N}$ agents participating in the distribution process $\left(\Sigma c_{i}-E\right) / N$, and the result represents the number of resources that each agent will loose given its initial maximum assignments.

To apply the principles of CEL to the mobile multi-channel assignment problem, this work proposes the definition of the Discrete CEL for Mobile radio resources distribution (DCELM) mechanism. DCELM follows the CEL distribution principles but has been designed to distribute resources of discrete nature (time slots for TDMA systems or codes for CDMA). It is important to note that only recently has been addressed the possibility to extend bankruptcy distribution rules to handle discrete resources [7]. However, the work reported in [7] considers utility values that are directly proportional to the number of assigned resources. On the other hand, this work considers a scenario where the relation between utility values and number of assigned resources is not proportional and varies depending on the service class. To the author's best knowledge, this work addresses then for the first time the use of bankruptcy policies to distribute discrete resources considering a variety of user profiles and requests. The CEL policy tries to reduce resources to each user given the use of utility values that are directly proportional to the number of assigned resources. On the other hand, the DCELM 
proposal is also aimed at trying to obtain equal losses for all users but considering utility values that are not directly proportional to the number of assigned radio resources. This novel approach provides a closer representation of an heterogeneous traffic scenario where each traffic type does not require the same number of resources to achieve the same QoS satisfaction level. The DCELM proposal is based on the normalized utility values reported in Fig. 1 and unassigns radio resources until the total number of assigned radio resources equals the number of available radio resources. Given each service class resources need, DCELM does not equally unassign resources to each user but unassigns resources to achieve equal utility values; to this end, the number of unassign resources might be different to each service class. Radio resources are removed from users with the highest utility values. When different users have the same utility value, the lower priority users loose first the assigned radio resource based on the following service priorities: $64 \mathrm{kbps}$ video (higher priority), 32kbps video, 16kbps video, web and email (lower priority).

\section{MAximum Utility Increase (MAUI)}

To provide a framework to which compare the performance and operation of the DCELM proposal, this work also defines the MAximum Utility Increase (MAUI) scheme. The MAUI proposal assigns each radio resource to the user that will experience the higher utility increase when assigned such radio resource. Consequently, the MAUI proposal is aimed at maximising the global utility values achievable with the available resources. However, it is important that since MAUI considers a sequential resource assignment process it might not provide the highest possible global utility value that could be achieved if all assignment combinations were computed. However, it provides a sub-optimal solution with a reduced computational cost that allows for it potential real implementation.

When applying the DCELM and MAUI proposals, all channels are re-distributed each time a user requests access to the system or a user ends its transmission. Only active realtime video users can maintain their assigned radio resources if those are not higher than the required number to guarantee their minimum QoS satisfaction level. If this is the case, these active users will only maintain the number of resources corresponding to the minimum QoS levels and the remaining resources will be redistributed among competing users. For both schemes, if several users are candidates to receive the same radio resource, these are served based on the previously defined service priorities.

\section{SIMULATION PLATFORM}

The performance of the proposed multi-channel assignment policies has been assessed by means of SPHERE (Simulation Platform for HEterogeneous wiREless systems), an advanced heterogeneous system level simulation platform [8]. The platform integrates three advanced system level simulators emulating the GPRS (General Packet Radio Service), EDGE, and HSDPA (High-Speed Downlink Packet Service) radio technologies. However, this work does not consider heterogeneous networks but is based instead on multi-slot assignments for EDGE. The work could, nevertheless, be extended to address the HSDPA multi-code assignment problem, and even the design of common radio resource management policies in $\mathrm{B} 3 \mathrm{G}$ heterogeneous systems.

SPHERE emulates email, WWW and H.263 real-time video transmissions at the packet level, which allows an accurate evaluation of the final user perceived QoS. The simulation platform implements all EDGE transmission modes and models its adaptive radio interface through the use of Link Adaptation (LA). This technique periodically selects the optimum transmission mode for the experienced channel quality conditions. For non real-time services, the transmission mode that maximises the throughput is selected. For real-time services, the algorithm proposed in [9] has been used. Erroneously received data is retransmitted by means of a selective ARQ protocol, for non real-time services; retransmission protocols are deactivated for delay-sensitive real-time services. Table II summarises the simulator's macrocellular downlink system configuration.

TABLE II

SySTEM LEVEL Simulation PARAMETERS

\begin{tabular}{|c|c|}
\hline Parameter & Value \\
\hline Simulated cells & 25 \\
Cluster size & 4 \\
Sectorisation & $120^{\circ}$ \\
Interfering cells & $1^{\mathrm{o}}$ and $2^{\circ}$ tiers co-channel cells \\
Cellular radio & $1 \mathrm{~km}$ \\
Channels per sector & $8(1$ carrier $)$ \\
Users per sector & $16: 5$ web, 5 email, 2 video $16 \mathrm{kbps}, 2$ \\
Mobility & video $32 \mathrm{kbps}, 2$ video $64 \mathrm{kbps}$ \\
Pathloss & $50 \mathrm{~km} / \mathrm{h}$ user speed \\
Shadowing & COST 231 Hata \\
Channel selection & Log-normal with 6dB standard deviation \\
Scheduling & Random \\
ARQ & First Come First Served \\
& Window size $=384$ RLC blocks \\
Link Adaptation & ARQ report each 32 RLC blocks \\
& LA updating period=100ms \\
\hline
\end{tabular}

\section{IV.PERFORMANCE EVALUATION}

The performance of the DCELM and MAUI proposals is compared with static multi-channel assignment schemes defined in Table III. The static assignment policies have been configured to request the resources requested to achieve minimum, mean or maximum user QoS satisfaction levels based on the utility values reported in Fig. 1. When applying a fixed allocation policy, if the number of resources requested by a user is not available, the user will be queued until enough resources are made available. The performance of the various multi-channel distribution policies is evaluated by means of the user satisfaction parameter, which accounts for the percentage of web pages or emails transmitted in less than 4 seconds and the percentage of video frames transmitted before the next frame is to be transmitted. 
TABLE III

RADIO RESOURCES FOR STATIC ASSIGNMENT POLICIES

\begin{tabular}{|c|c|c|c|c|c|}
\hline & Web & Email & $\begin{array}{c}\text { 16kbps } \\
\text { video }\end{array}$ & $\begin{array}{c}\text { 32kbps } \\
\text { video }\end{array}$ & $\begin{array}{c}\text { 64kbps } \\
\text { video }\end{array}$ \\
\hline Min QoS & 2 & 1 & 1 & 2 & 4 \\
\hline Mean QoS & 3 & 2 & 2 & 4 & 7 \\
\hline Max QoS & 6 & 3 & 4 & 8 & 8 \\
\hline
\end{tabular}

Tables IV and V report the user satisfaction levels that can be achieved with fixed policies and the DCELM and MAUI proposals under the operating conditions defined in Table II. First of all, it is important to note the static multi-channel assignment policies' inefficiency to cope with the user QoS demands, in particular for the most demanding services. On the other hand, the DCELM proposal achieves the higher user satisfaction levels for the most demanding services, that is real-time video users. Table VI shows that nearly all simulated 32 and $64 \mathrm{kbps}$ video transmissions were assigned radio resources at each channel distribution round. This percentage is reduced as the channel resources demand increases and the service priority is reduced. This is the case because the DCELM proposal initially assigns to all users the resources corresponding to their maximum demand and then unassign resources to users experiencing the higher utility values. With DCELM, the users with higher probabilities to retain radio resources are those that achieve lower utility values with those resources. Also, when users that require more than one radio resource to achieve a non null utility value are unassigned one of these resources they will obtain a null utility value. Following the DCELM principle, these users will not be unassigned the remaining resources until the rest of users experience a null utility value. While this behaviour will result in a more spread channel distribution, such distribution does not guarantee to achieve the minimum QoS levels. Also, users requiring only one radio resource will be those that first loose the assigned resources, which partly explains the poor performance for email and 16kbps video users. With DCELM, users can also receive a number of resources below to their minimum QoS threshold in high system loads, such as those emulated in this paper and resulting in low user throughputs and increased transmission durations. High transmission durations result in a high number of competing users at each channel distribution round. As a result, lower prioritised services (such as email) are rarely served.

To overcome the original DCELM limitations, a modified version has been implemented to optimise the use of radio resources assigned to users requiring more than one channel to achieve a non null utility value. The modified DCELM's operation is similar to the original DCELM, except that when resources need to be unassign to a user experiencing its minimum utility value instead of unassigning only one resource following the original DCELM process, all resources are unassigned and distributed users. With this approach, instead of sparsely assigning resources to competing users, resources are assigned trying to at least always guarantee the minimum user QoS satisfaction level. In fact, avoiding the assignment of radio resources that don't guarantee the minimum QoS levels, results in a better, and more uniform,
QoS performance for the most demanding users (Table V). The improved modified DCELM behaviour with regards to DCELM is due to the fact that the modified DCELM approach assigns a number of radio resources per service class that better matches the minimum user demands reported in Fig. 1. The results shown in Tables IV and VI actually prove that the modified DCELM proposal obtains higher real-time satisfaction levels despite serving a lower percentage of realtime users, which proves the important effect of the implemented multi-channel distribution policies.

TABLE IV

STATIC POLICIES USER SATISFACTION (\%)

\begin{tabular}{|c|c|c|c|}
\hline & Min QoS & Mean QoS & Max QoS \\
\hline Web & 79.38 & 79.99 & 76.32 \\
\hline Email & 66.05 & 77.22 & 70.59 \\
\hline 16kbps video & 74.88 & 81.11 & 43.42 \\
\hline 32kbps video & 57.17 & 38.22 & 23.20 \\
\hline 64kbps video & 25.93 & 9.93 & 22.11 \\
\hline
\end{tabular}

TABLE V

DCELM AND MAUI USER SATISFACTION (\%)

\begin{tabular}{|c|c|c|c|}
\hline & DCELM & Mod. DCELM & MAUI \\
\hline Web & 57.48 & 53.20 & 45.01 \\
\hline Email & 15.09 & 14.90 & 91.12 \\
\hline 16kbps video & 63.71 & 86.25 & 99.94 \\
\hline 32kbps video & 79.64 & 87.41 & 81.79 \\
\hline 64kbps video & 83.65 & 87.23 & 85.48 \\
\hline
\end{tabular}

The results illustrated in Tables V and VIII show that the MAUI proposal can significantly improve the performance except for web services. This is due to the fact that the MAUI allocation process is quite conditioned by the defined utility functions. At the beginning of each distribution round most users will begin with an utility value equal to zero, except for previously active video users that maintain their assigned radio resources. MAUI will then allocate each resource to the user that will experience higher utility increases. Consequently, users requiring only one channel to obtain a non-null utility value (minimum QoS level) are those that are firstly served. This explains the improved MAUI email and $16 \mathrm{kbps}$ video performance. After email and $16 \mathrm{kbps}$ video users are served, users are assigned resources following the previously established service priorities, which explains the web poorer performance. For such services, a relaxed QoS satisfaction definition could significantly improve the performance statistics given that $62.09 \%$ of web transmissions could be handled less than 6 seconds and $72.33 \%$ in less than 8 seconds. Shown in Table VIII, MAUI tends to allocate very high number of resources to very few simultaneous active users. Although this might result in a less instantaneously fair distribution, what is important is not that resources are fairly distributed but that user perceived QoS is fairly distributed. By assigning a significant amount of resources to a single user, MAUI increases the user's throughput and reduces its transmission time, which allows quickly freeing the resources and burstly serving a new user. 
TABLE VI

DCELM PERFORMANCE

\begin{tabular}{|c|c|c|c|c|c|c|}
\hline & $\begin{array}{c}\text { Mean } \\
\text { through } \\
\text { put (kbps) }\end{array}$ & $\begin{array}{c}\text { Mean } \\
\text { waiting } \\
\text { time (s) }\end{array}$ & $\begin{array}{c}\mathbf{n}^{\mathbf{0}} \text { of } \\
\text { assigned } \\
\text { slots }\end{array}$ & $\begin{array}{c}\text { \% of } \\
\text { aborted } \\
\text { frames }\end{array}$ & $\begin{array}{c}\text { \% of } \\
\text { unsent } \\
\text { frames }\end{array}$ & $\begin{array}{c}\text { \% of } \\
\text { served } \\
\text { users }\end{array}$ \\
\hline Web & 37.31 & 2.3355 & 1.34 & - & - & 67.50 \\
\hline Email & 34.10 & 84.8155 & 1.11 & - & - & 7.76 \\
\hline $\begin{array}{c}\text { 16kbps } \\
\text { video }\end{array}$ & 24.87 & 0.0529 & 1.04 & 13.60 & 22.69 & 56.55 \\
\hline $\begin{array}{c}32 \mathrm{kbps} \\
\text { video }\end{array}$ & 36.07 & 0.0075 & 1.62 & 19.88 & 0.49 & 96.91 \\
\hline $\begin{array}{c}\text { 64kbps } \\
\text { video }\end{array}$ & 73.93 & 0.0069 & 3.31 & 16.33 & 0.02 & 99.68 \\
\hline
\end{tabular}

TABLE VII

MODIFIED DCELM PERFORMANCE

\begin{tabular}{|c|c|c|c|c|c|c|}
\hline & $\begin{array}{c}\text { Mean } \\
\text { through } \\
\text { put (kbps) }\end{array}$ & $\begin{array}{c}\text { Mean } \\
\text { waiting } \\
\text { time (s) }\end{array}$ & $\begin{array}{c}\mathbf{n}^{\mathbf{0}} \text { of } \\
\text { assigned } \\
\text { slots }\end{array}$ & $\begin{array}{c}\text { \% of } \\
\text { aborted } \\
\text { frames }\end{array}$ & $\begin{array}{c}\text { \% of } \\
\text { unsent } \\
\text { frames }\end{array}$ & $\begin{array}{c}\text { \% of } \\
\text { served } \\
\text { users }\end{array}$ \\
\hline Web & 54.37 & 5.1228 & 1.87 & - & - & 38.46 \\
\hline Email & 34.48 & 87.6210 & 1.14 & - & - & 6.77 \\
\hline $\begin{array}{c}\text { 16kbps } \\
\text { video }\end{array}$ & 24.93 & 0.0167 & 1.05 & 10.77 & 11.22 & 86.86 \\
\hline $\begin{array}{c}32 \mathrm{kbps} \\
\text { video }\end{array}$ & 46.22 & 0.0132 & 2.02 & 14.34 & 2.98 & 91.11 \\
\hline $\begin{array}{c}\text { 64kbps } \\
\text { video }\end{array}$ & 83.50 & 0.0085 & 3.72 & 1.23 & 0.12 & 97.41 \\
\hline
\end{tabular}

TABLE VIII

MAUI PERFORMANCE

\begin{tabular}{|c|c|c|c|c|c|c|}
\hline & $\begin{array}{c}\text { Mean } \\
\text { through } \\
\text { put (kbps) }\end{array}$ & $\begin{array}{c}\text { Mean } \\
\text { waiting } \\
\text { time (s) }\end{array}$ & $\begin{array}{c}\mathbf{n}^{\circ} \text { of } \\
\text { assigned } \\
\text { slots }\end{array}$ & $\begin{array}{c}\text { \% of } \\
\text { aborted } \\
\text { frames }\end{array}$ & $\begin{array}{c}\text { \% of } \\
\text { unsent } \\
\text { frames }\end{array}$ & $\begin{array}{c}\% \text { of } \\
\text { served } \\
\text { users }\end{array}$ \\
\hline Web & 228.08 & 11.0277 & 7.99 & - & - & 4.85 \\
\hline Email & 178.79 & 0.0071 & 6.65 & - & - & 99.99 \\
\hline $\begin{array}{c}\text { 16kbps } \\
\text { video }\end{array}$ & 67.37 & 0.0069 & 4.91 & 0.06 & 2.21 & 99.96 \\
\hline $\begin{array}{c}\text { 32kbps } \\
\text { video }\end{array}$ & 129.80 & 0.0437 & 7.39 & 2.28 & 0.01 & 40.51 \\
\hline $\begin{array}{c}\text { 64kbps } \\
\text { video }\end{array}$ & 150.23 & 0.0292 & 7.45 & 13.93 & 12.55 & 57.50 \\
\hline
\end{tabular}

Despite improving the performance over DCELM, the MAUI proposal can provide a lower user QoS satisfaction for the most demanding services compared to the modified DCELM approach as shown in Fig. 2. In fact, Fig. 2 shows that while the modified DCELM approach provides uniform user satisfaction levels among the higher priority services independently of the system's load, the MAUI performance is highly influenced by the system load distribution.

\section{CONCLUSIONS}

Bankruptcy and utility-based multi-channel assignment policies have been proposed and evaluated under heterogeneous traffic conditions. The proposed techniques are aimed at efficiently using the available radio resources by considering the varying resources demand per traffic class. The DCELM and MAUI proposals significantly improve the system performance, highlighting the importance of establishing adequate channel assignment policies based on overall system objectives and the potential of the bankruptcy techniques to address radio resource management problems. While DCELM tends to provide homogeneous performance independently of the system load, MAUI provides the higher performance under certain system loads.
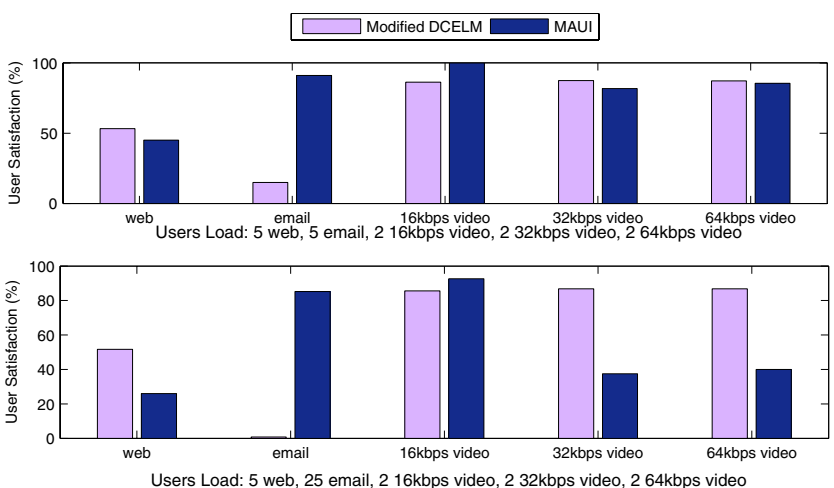

Fig. 2. User satisfaction (\%) for new users load.

\section{ACKNOWLEDGMENT}

This work has been supported by the Ministry of Education and Science (Spain) and FEDER funds under the projects TEC2005-08211-C02-02 and MTM2005-09184-C02-02, and by the Generalitat Valenciana under the project ACOMP07/256.

\section{REFERENCES}

[1] E. Vivier, M. Terré, B. Fino, "Determination of all possible resource allocations in a packet communication network", in Proc. of the IEEE Vehicular Technology Conference, September 2002, pp. 2346-2350.

[2] A. Kuurne, D. Fernández, R. Sánchez, "Service Based Prioritization in (E)GPRS Radio Interface", in Proc. of the IEEE Vehicular Technology Conference, September 2004, pp. 2625-2629.

[3] A. Malla, M. El-Kadi, S. Olariu, P. Todorova, "A Fair Resource Allocation Protocol for Multimedia Wireless Networks", IEEE Transactions on Parallel and Distributed Systems, vol.14, pp. 63-71, January 2003.

[4] M. Dramatinos, G. D. Stamoulis, C. Courcoubetis, "Auction-based Resource Allocation in UMTS High Speed Downlink Packet Access (HSDPA)", in Proc. of the Next Generation Internet Networks Conference, 18-20 April 2005, pp. 434-441.

[5] W. Thomson, "Axiomatic and game-theoretic analysis of bankruptcy and taxation problems: a survey", Mathematical Social Sciences, vol 45, pp 249-297, 2003.

[6] 3GPP, Services and service capabilities, 3GPP TS 22.105, version 6.3.0, 2005.

[7] C. Herrero and R. Martinez, "Balanced Allocation Methods for Claims Problems with Indivisibilities", CORE Discussion Paper 2006/66, July 2006. Available at SSRN: http://ssrn.com/abstract=944416

[8] M. López-Benítez, M. Lucas-Estañ and J. Gozalvez, "A dynamic radio simulation platform for the study of radio resource management techniques in heterogeneous wireless systems", in Proc. of the 9th ACM/IEEE MSWIM conference (Demos Session), October 2006.

[9] J. Gozálvez, M. López-Benítez and O. Lázaro, "Link adaptation algorithm for improved wireless transmission of delay-sensitive packet data services", Electronic Letters, pp 813-815, July 2005 